\title{
Review
}

\section{The promise and potential hazards of adenovirus gene therapy}

Gene therapy has been heralded as the "medicine of the 21 st century". With this has come an expectation which has yet to be met by experience. Thus the past decade has seen some 4000 patients entered into a variety of gene therapy trials with very few documented successes. Much of the "hype and hope" surrounding clinical gene therapy reflects the gap between the need to carefully perform phase I clinical trials to assess toxicity and tolerability and the public expectation that this approach is the panacea to chronic disease. Recent publicity highlights the current dilemma. The death in the USA of an 18 year old with ornithine transcarbamylase (OTC) deficiency after intrahepatic arterial injection of an adenovirus vector carrying a wild-type version of the defective enzyme has precipitated a flurry of reports and congressional hearings focusing on the ethics of such trials and on the very nature of clinical research itself. ${ }^{1}$ In contrast, a recent report provides one of the first clear cut gene therapy successes - the reconstitution of immune responses in two children with the SCID-X1 disorder by retroviral delivery of the wild-type gene to bone marrow. ${ }^{2}$ These two examples are interesting in that the therapies target rare monogenic diseases where returning the wild-type gene is the only rational therapeutic option while also posing the most rigorous test of the gene therapy approach - the ability to provide long term correction of gene defects. It is however in the area of the common ills such as heart disease and cancer that gene therapy promises so much and, despite current concerns, there is no doubt that with the necessary technical developments gene therapy will significantly improve the treatment of the major contemporary causes of premature mortality.

It is generally accepted that the major impediment to the successful application of gene therapy for the treatment of a range of diseases is not a paucity of therapeutic genes but the lack of an efficient non-toxic gene delivery system. While approaches using synthetic vectors are being developed, ${ }^{3}$ the most efficient gene delivery systems appropriate for clinical application which are currently available are based on virus vectors. Having evolved to deliver their genes to target cells, viruses can be easily manipulated to express therapeutic genes and the choice of virus type depends largely on the target cell and on the requirement for either transient or long term transgene expression. Virus vectors currently in use include adenovirus, adeno associated virus, herpes simplex virus, retrovirus, and lentivirus. Of these, the ability of adenoviruses to efficiently infect and deliver genes to a range of cells and to be generated to high titres has led to their widespread application. The commonly used first generation recombinant adenovirus vectors are based on adenovirus type 5, which causes mild respiratory infection in humans. ${ }^{4}$ They have been modified by deletion of the E1 region, which encodes proteins that regulate expression of the other early genes (as well as the late virus structural proteins), thereby rendering virus replication defective. It was envisaged that the inability of these recombinant adenoviruses to replicate efficiently would prevent the production of unwanted viral proteins by infected cells, thus limiting both direct adenovirus toxicity and possible harmful consequences of antiadenovirus immune responses. In practice this has not always been the case and studies in rodents, primates, and humans have provided variable results, highlighting the need for a more detailed understanding of the natural history of adenovirus infection in humans and questioning the value of animal models in determining the safety of virus vectors. In this review we will consider the nature of adenovirus toxicity and the development of improved adenovirus vectors.

\section{What's toxic about adenovirus vectors?}

Much concern has focused on the direct toxic effects of adenoviruses, particularly as intravenous administration of the virus can induce acute liver injury, as shown in animal models. It is this effect which may have triggered the cascade of events leading to the death of the patient with OTC deficiency - in this case the recombinant virus was injected directly into the hepatic artery. Studies in mice have highlighted the dose limiting liver toxicity of intravenously administered virus, which in this model is mainly due to an acute inflammatory response involving the release of certain cytokines (interleukin 6 , interleukin 8 , tumour necrosis factor $\alpha$ ) and the recruitment of immune effector cells into the liver. ${ }^{5-7}$ These effects are manifest within the first few hours of adenovirus administration and do not require de novo virus gene expression. A recent study demonstrated that adenovirus induced chemokine gene expression within the liver occurs within one hour after infection and results in the recruitment of neutrophils which are principally responsible for the hepatic injury. ${ }^{8}$ The ability of replication defective adenovirus to induce chemokine expression appears to be a consequence of the interaction of the virus capsid with target cells, although the precise mechanism by which virus binding elicits induction of gene expression remains to be determined. Hepatotoxicity together with prolongation of thrombin clotting time has also been observed in rhesus macaques receiving high doses of intravenous adenovirus. ${ }^{9}$ Interestingly, the acute liver toxicity resolved except for persistent hypofibrinogenaemia in the high dose recipient. Another disturbing aspect of this study was the highly immunogenic nature of the transgene product (human coagulation factor IX), which is in stark contrast with its lack of effects when administered to the same monkeys as a purified protein. This raises the possibility that therapeutic genes expressed within the context of a recombinant adenovirus may stimulate immune responses, thereby compromising their therapeutic utility. However, another study in which rhesus monkeys were repeatedly infected by bronchoscopic instillation with high doses of a second generation (E1 and E4 deleted) adenovirus vector carrying the cystic fibrosis transmembrane conductance regulator (CFTR) gene demonstrated generation of both humoral and cell mediated immune responses to the virus but not to the

Abbreviations used in this paper: CFTR, cystic fibrosis transmembrane conductance regulator; CAR, coxsackie-adenovirus receptor; CTL, cytotoxic T lymphocyte; FGF, fibroblast growth factor; OTC, ornithine transcarbamylase; 
CFTR transgene. ${ }^{10}$ A major consequence of the antiadenovirus immune response was the marked reduction of transgene expression following multiple dosing, which appears to result mainly from stimulation of neutralising antibody responses although adenovirus specific cytotoxic $\mathrm{T}$ lymphocytes (CTLs) were also detected.

It is clear from previous studies that intravenous injection of recombinant adenovirus is associated with rapid dose related liver toxicity. However, low doses of E1 deleted viruses can be used with minimal toxicity even in animals with damaged livers. Thus Nakatani and colleagues ${ }^{11}$ demonstrated that adenovirus mediated gene delivery can be achieved efficiently and safely in mice with either liver cirrhosis or fulminant hepatitis. Intravenous administration of low doses of a $\beta$ galactosidase expressing E1 deleted adenovirus resulted in some differences in gene transduction efficiency when comparing normal mice with those with either cirrhosis or hepatitis, but no increased toxicity or differences in antiadenovirus immune responses were observed. These data are encouraging and suggest that adenovirus directed gene delivery may be useful in patients with severe liver disease provided that low level transgene expression is sufficient to obtain a therapeutic benefit. The problem with such studies is that they are mostly performed in rodents, and that these models are unlikely to reflect the behaviour of recombinant adenovirus in humans. Thus factors determining the ability of human tissues (including the liver) to be infected by adenovirus and the relative importance of the coxsackie-adenovirus receptor (CAR) and $\alpha \mathrm{v}$ integrins in mediating virus uptake in different tissues remains unknown. ${ }^{12} \mathrm{~A}$ major concern is the inappropriate use of rodent models both for investigating immune responses to (wild-type) adenovirus infections and for preclinical evaluation of adenovirus based gene therapy. Thus most rodents are not fully permissive for adenovirus replication, and the major CTL response in these animals is directed against virus early antigens. ${ }^{13-16}$ In contrast, data from adenovirus infected healthy humans highlights the role of virus capsid antigens as immune targets. ${ }^{17}{ }^{18}$ Pre-existing immune responses in humans may also limit the use and effectiveness of recombinant adenovirus and cannot be properly assessed in rodent models. Nevertheless, experiments in animals have served to emphasise the transient nature of adenovirus delivered transgene expression in the presence of an immune response (particularly neutralising antibodies) and have led to attempts to modify the vector either by deleting further virus genes or by constructing recombinant adenoviruses based on the less common adenovirus serotypes. ${ }^{19} 20$ The impact of pre-existing adenovirus immunity on toxicity, tolerability, and efficacy of recombinant adenovirus gene therapy in cancer patients has only been documented in a few reports. GaherySegard et al (1997) $)^{21}$ demonstrated elevation in both CD4+ve proliferative and CTL responses to adenovirus in four lung carcinoma patients who received a single intratumoural injection of a recombinant adenovirus expressing $\beta$ galactosidase. A group studying the effect of thymidine kinase/ganciclovir gene therapy in patients with malignant mesothelioma found evidence of both local and systemic inflammatory responses subsequent to intrapleural injection of recombinant adenovirus ${ }^{22}$ and reported significant increases in proliferative $\mathrm{T}$ cell responses after treatment. ${ }^{23}$ Thus studies aimed at gaining a more detailed understanding of the natural history of adenovirus infection in humans and of the nature of adenovirus specific immunity are clearly warranted.

\section{Modification of adenoviruses for gene therapy}

The toxicity of the first generation E1 deleted adenoviruses is a consequence of both antivirus immune responses and the broad tropism of adenovirus infection. Thus attempts to modify the behaviour of the virus are a focus of much interest, and concentrate on removal of additional adenoviral genes and on modifying the tropism of the virus. Given the adverse effects of both acute and chronic immune responses, a number of approaches aimed at controlling host responses have been examined. Blocking cell adhesion and immune costimulatory molecules at the time of adenovirus infection can reduce the toxic response and diminish immune responses, allowing successful readministration. ${ }^{24}{ }^{25}$ An alternative approach is based on cytokine treatment (for example interleukin 12) to alter the balance of helper $\mathrm{T}$ cells, thereby skewing the antiviral response to either antibody production or cell mediated cytotoxicity. ${ }^{26}$ Somewhat paradoxically, certain adenovirus encoded genes can be used to downregulate immune responses. Thus the function of the E3 encoded gp $19 \mathrm{~K}$ protein is to inhibit the transport of MHC class I molecules to the cell surface and thus to reduce induction and activity of the CTL response. ${ }^{27-30}$ Expression of gp $19 \mathrm{~K}$ has been shown to increase the persistence of transgene expression in an E1+E3 deleted vector. ${ }^{31}{ }^{32}$ Another set of E3 encoded proteins (RID, E3-14.7K) inhibit apoptosis induced by tumour necrosis factor $\alpha$, CD95L, and TRAIL and could also be used to downregulate the acute inflammatory response as well as cell mediated immunity against recombinant adenoviruses. ${ }^{33-35}$

The ability of E1 deleted adenovirus to replicate under certain conditions and the associated expression of viral genes which are either cytotoxic or elicit host immunity has led to the development of virus vectors with increasingly deleted genomes. Removal of viral genes encoding key replication functions (E2 encoded DNA polymerase, DNA binding protein, and the genome associated terminal protein) or important regulatory functions (for example, E4 proteins) has led to the production of recombinant viruses with a reduced ability to stimulate immune responses while achieving sustained transgene expression. ${ }^{36-38}$ An extreme example of this approach is the development of "gutless" viruses devoid of all adenovirus genes but retaining the sequences essential for replication and packaging of the genome. ${ }^{39-43}$ These viruses allow for the introduction of up to $35 \mathrm{~kb}$ of DNA but require a helper virus for replication, making downstream processing of pure recombinant virus for clinical studies more difficult. However, the development of packaging defective helper viruses which make use of the site specific cre-lox bacterial recombination system to discriminate helper from vector offer the promise of a resolution to this problem. ${ }^{44-46}$

Altering the adenovirus coat to evade or minimise the effect of neutralising antibodies or to enable re-targeting of the virus to specific cell types is an area of much interest being actively pursued in both academia and the pharmaceutical industry. Attempts to circumvent the preexisting humoral response and to alter the virus tropism have been made using chimeric capsid proteins. Thus a virus with a chimeric adenovirus $5 / 12$ hexon, where the surface loops specifying type specific neutralisation came from adenovirus 12, was found to overcome virus neutralisation in mice primed with adenovirus 5 virus. ${ }^{47} \mathrm{~A}$ chimeric fibre with the receptor binding knob domain of adenovirus 3, which infects cells through an unknown receptor, distinct from CAR, was used to produce an adenovirus $5 / 3$ vector resulting in an adenovirus with altered tropism. ${ }^{48}$ Initial attempts to redirect adenovirus infection have used bi-specific molecules to attach the virus to specific cell surface receptors. In this approach the 
natural tropism of the virus is blocked by the use of a neutralising antibody to the fibre which is attached either by conjugation or by genetic engineering to a targeting ligand or a cell type specific antibody. Thus adenovirus infection has been redirected to the folate receptor by conjugating folate to the Fab fragment of a neutralising antifibre monoclonal antibody ${ }^{49}$ and to the fibroblast growth factor (FGF) receptor by use of an antibody-FGF complex. ${ }^{50} \mathrm{~A}$ more refined strategy is to genetically modify the fibre or hexon to incorporate ligands (peptides, short chain antibodies) which alter the tropism of the virus..$^{51-55}$ While initial studies in this area have generated modified vectors with extended tropism, the challenge has been to identify the CAR binding site on fibre and replace this with a targeting ligand. Identification of a conserved receptor binding site on the fibre protein of those adenoviruses which recognise $\mathrm{CAR}^{56}$ and the publication of the crystal structure of the adenovirus 12 fibre knob domain in complex with the binding domain I of $\mathrm{CAR}^{57}$ allows for more precise engineering of fibre for various re-targeting strategies. Key amino acids within the fibre essential for CAR binding have been defined, ${ }^{58}$ which allows the design of fibre mutants which ablate CAR binding and incorporate an alternative ligand, thus realising the aim of redirecting virus binding. Strategies to allow production of viruses that lack CAR binding capacity have also been developed, ${ }^{59}{ }^{60}$ and the stage is now set for the design and clinical application of genetically modified adenovirus vectors with specific targeting attributes.

L S YOUNG

CRC Institute for Cancer Studies, V MAUTNER

University of Birmingham,

Birmingham B15 2TA, UK

Correspondence to: L S Young. L.S.Young@bham.ac.uk

1 Marshall E. Biomedicine-Gene therapy on trial. Science 2000;288:951-7. 2 Cavazzana-Calvo M, Hacein-Bey S, Basile CD, et al. Gene therapy of human severe combined immunodeficiency (SCID)-X1 disease. Science 2000;288:669-72.

3 Lollo PC, Banaszczyk MG, Chiou HC. Obstacles and advances in non-viral gene delivery. Curr Opin Mol Ther 2000;2:136-42.

4 Horwitz MS. Adenoviruses. In: Fields BN, Knipe DM, eds. Virology, 2nd

4 Horwitz MS. Adenoviruses. In: Fivelogy,
edn. New York: Raven Press, 1990:1723-40.
5 Duncan SJ, Gordon FCA, Gregory DW, et al. Infection of mouse liver by Duncan SJ, Gordon FCA, Gregory DW, et al. Infection
human adenovirus type 5. f Gen Virol 1978;40:45-61.

6 Gooding L. Regulation of TNF-mediated cell death and inflammation by 6 Gooding L. Regulation of TNF-mediated cell death

7 Worgall S, Wolff G, FalckPedersen E, et al. Innate immune mechanisms dominate elimination of adenoviral vectors following in vivo administration. Hum Gene Ther 1997;8:37-44.

8 Muruve DA, Barnes MJ, Stillman IE, et al. Adenoviral gene therapy leads to rapid induction of multiple chemokines and acute neutrophil-dependent hepatic injury in vivo. Hum Gene Ther 1999;10:965-76.

9 Lozier JN, Metzger ME, Donahue RE, et al. Adenovirus-mediated expression of human coagulation factor IX in the rhesus macaque is associated with dose-limiting toxicity. Blood 1999;94:3968-75.

10 Kaplan JM, St George JA, Pennington SE, et al. Humoral and cellular immune responses of nonhuman primates to long-term repeated lung exposure to Ad2/CFTR-2. Gene Ther 1996;3:117-27.

11 Nakatani T, Kuriyama S, Tominaga K, et al. Assessment of efficiency and safety of adenovirus-mediated gene transfer into normal and damaged murine livers. Gut 2000;47:563-70.

12 Bergelson JM. Receptors mediating adenovirus attachment and internalization. Biochem Pharmacol 1999;57:975-9.

13 Rawle FC, Knowles BB, Ricciardi RP, et al. Specificity of the mouse cytotoxic lymphocyte-T response to adenovirus 5-E1a is immunodominant in $\mathrm{h}-2 \mathrm{~b}$, but not in h-2d or h-2k mice. F Immunol 1991;146:3977-84.

14 Routes JM, Bellgrau D, McGrory WJ, et al. Anti-adenovirus type 5 cytotoxic lymphocyte T-immunodominant epitopes are encoded by the E1 a gene. $\mathcal{F}$ Virol 1991;65:1450-7.

15 Pereira DS, Rosenthal KL, Graham FL. Identification of adenovirus E1a regions which affect MHC class-I expression and susceptibility to cytotoxic T-lymphocytes. Virology 1995;211:268-77.

16 Sijts AJAM, Standera S, Toes REM, et al. MHC class I antigen processing of an adenovirus CTL epitope is linked to the levels of immunoproteasomes in infected cells. F Immunol 2000;164:4500-6.

17 Flomenberg P, Piaskowski V, Truitt RL, et al. Characterization of human proliferative T-cell responses to adenovirus. F Infect Dis 1995;171:1090-6.

18 Smith CA, Woodruff LS, Kitchingman GR, et al. Adenovirus-pulsed dendritic cells stimulate human virus-specific T-cell responses in vitro. $\mathcal{f}$ Virol 1996;70:6733-40.

19 Yang YP, Nunes FA, Berencsi K, et al. Cellular immunity to viral antigens limits E1-deleted adenoviruses for gene therapy. Proc Natl Acad Sci USA 1994;91:4407-11.
20 Mastrangeli A, Harvey BG, Yao J, et al. Sero-switch adenovirus-mediated in-vivo gene-transfer-circumvention of anti-adenovirus humoral immune defenses against repeat adenovirus vector administration by changing the adenovirus serotype. Hum Gene Ther 1996;7:79-87.

21 Gahery-Segard H, Molinier-Frenkel V, LeBoulaire C, et al. Phase I trial of recombinant adenovirus gene transfer in lung cancer-Longitudinal study of the immune responses to transgene and viral products. $\mathcal{F}$ Clin Invest 1997;100:2218-26.

22 Sterman DH, Treat J, Litzky LA, et al. Adenovirus-mediated herpes simplex virus thymidine kinase/ganciclovir gene therapy in patients with localized malignancy: Results of a phase I clinical trial in malignant mesothelioma. Hum Gene Ther 1998;9:1083-92.

23 MolnarKimber KL, Sterman DH, Chang M, et al. Impact of preexisting and induced humoral and cellular immune responses in an adenovirus-based gene therapy phase I clinical trial for localized mesothelioma. Hum Gene Ther 1998;9:2121-33.

24 Yang Y, Su Q, Grewal IS, et al. Transient subversion of CD40 ligand function diminishes immune responses to adenovirus vectors in mouse liver and tion diminishes immune responses to ade
lung tissues. F Virol 1996;70:6370-7.

25 Wilson CB, Embree LJ, Schowalter D, et al. Transient inhibition of CD28 and $\mathrm{CD} 40$ ligand interactions prolongs adenovirus-mediated transgene expression in the lung and facilitates expression after secondary vector administration. $\mathcal{F}$ Virol 1998;72:7542-50.

26 Yang YP, Trinchieri G, Wilson JM. Recombinant IL-12 prevents formation of blocking IgA antibodies to recombinant adenovirus and allows repeated gene therapy to mouse lung. Nat Med 1995;1:890-3.

27 Burgert HG, Kvist S. An adenovirus type-2 glycoprotein blocks cell-surface expression of human histocompatibility class-I antigens. Cell 1985;41:98797.

28 Rawle FC, Tollefson AE, Wold WSM, et al. Mouse anti-adenovirus cytotoxic T lymphocytes. Inhibition of lysis by E3 gp19K but not E3 14.7K. F Immunol 1989;143:2031-7.

29 Ginsberg HS, Lundholm-Beauchamp U, Horswood RL, et al. Role of early region 3 (E3) in pathogenesis of adenovirus disease. Proc Natl Acad Sci USA 1989;86:3823-7.

30 Beier DC, Cox JH, Vining DR, et al. Association of human class I MHC alleles with the adenovirus E3/19K protein. F Immunol 1994;152:3862-72.

31 Bruder JT, Jie T, McVey DL, et al. Expression of gp19K increases the persistence of transgene expression from an adenovirus vector in the mouse lung and liver. $\mathcal{F}$ Virol 1997;71:7623-8.

32 Ilan Y, Droguett G, Chowdhury NR, et al. Insertion of the adenoviral E3 region into a recombinant viral vector prevents antiviral humoral and cellular immune responses and permits long-term gene expression. Proc Natl Acad Sci USA 1997;94:2587-92.

33 Tollefson A, Hermiston TW, Lichtenstein DL, et al. Forced degradation of Fas inhibits apoptosis in adenovirus-infected cells. Nature 1998;392:72630

34 Wold WSM, Tollefson AE. Adenovirus E3 proteins: 14.7K, RID, and gp19K inhibit immune-induced cell death; Adenovirus death protein promotes cell death. Semin Virol 1998;8:515-23.

35 Wold WSM, Doronin $\mathrm{K}$, Toth $\mathrm{K}$, et al. Immune responses to adenoviruses: viral evasion mechanisms and their implications for the clinic. Curr Opin Immunol 1999;11:380-6.

36 Amalfitano A, Hauser MA, Hu HM, et al. Production and characterization of improved adenovirus vectors with the E1, E2b, and E3 genes deleted. $\mathcal{F}$ Virol 1998;72:926-33.

37 Gao G-P, Yang Y, Wilson JM. Biology of adenovirus vectors with E1 and e4 deletions for liver-directed gene therapy. 7 Virol 1996;70:8934-43.

38 Lusky $M$, Christ M, Rittner K, et al. In vitro and in vivo biology of recombinant adenovirus vectors with E1, E1/E2A, or E1/E4 deleted. F Virol 1998; 72:2022-32.

39 Schiedner G, Morral N, Parks RJ, et al. A high capacity adenovirus vector with all viral genes deleted results in improved in vivo expression and decreased toxicity. Cancer Gene Ther 1997;4:328.

40 Kochanek S, Clemens PR, Mitani K, et al. A new adenoviral vector: Replacement of all viral coding sequences with $28 \mathrm{~kb}$ of DNA independently expressing both full-length dystrophin and betaindependently expressing both full-length dystroph

41 Chen HH, Mack LM, Kelly R, et al. Persistence in muscle of an adenoviral vector that lacks all viral genes. Proc Natl Acad Sci USA 1997;94:1645-50.

42 Morsy MA, Gu MC, Motzel S, et al. An adenoviral vector deleted for all viral coding sequences results in enhanced safety and extended expression of a leptin transgene. Proc Natl Acad Sci USA 1998;95:7866-71.

43 Lieber A, He C-Y, Kirillova I, et al. Recombinant adenoviruses with large deletions generated by cre-mediated excision exhibit different biological properties compared with first-generation vectors in vitro and in vivo. $\mathcal{f}$ Virol 1996;70:8944-60.

44 Parks RJ, Graham FL. A helper-dependent system for adenovirus vector production helps define a lower limit for efficient DNA packaging. $\mathcal{F}$ Virol 1997;71:3293-8

45 Parks RJ, Chen L, Anton M, et al. A helper-dependent adenovirus vector system: Removal of helper virus by Cre-mediated excision of the viral packaging signal. Proc Natl Acad Sci USA 1996;93:13565-70.

46 Benihoud K, Yeh P, Perricaudet M. Adenovirus vectors for gene delivery. Curr Opin Biotechnol 1999;10:440-7.

47 Roy S, Shirley PS, McClelland A, et al. Circumvention of immunity to the adenovirus major coat protein hexon. $\mathcal{7}$ Virol 1998;72:6875-9.

48 Krasnykh VN, Mikheeva GV, Douglas JT, et al. Generation of recombinant adenovirus vectors with modified fibers for altering viral tropism. $\mathcal{F}$ Virol 1996;70:6839-46.

49 Douglas JT, Rogers BE, Rosenfeld ME, et al. Targeted gene delivery by tropism-modified adenoviral vectors. Nat Biotechnol 1996;14:1574-8.

50 Goldman CK, Rogers BE, Douglas JT, et al. Targeted gene delivery to Kaposi's sarcoma cells via the fibroblast growth factor receptor. Cancer Res 1997;57:1447-51.

51 Michael SI, Hong JS, Curiel DT, et al. Addition of a short peptide ligand to the adenovirus fiber protein. Gene Ther 1995;2:660-8.

52 Dmitriev I, Krasnykh V, Miller CR, et al. An adenovirus vector with genetically modified fibers demonstrates expanded tropism via utilization of a coxsackievirus and adenovirus receptor-independent cell entry mechanism. f Virol 1998;72:9706-13.

53 Wickham TJ, Tzeng E, Shears LL, et al. Increased in vitro and in vivo gene transfer by adenovirus vectors containing chimeric fiber proteins. 7 Virol $1997 ; 71: 8221-9$ 
54 Vigne E, Mahfouz I, Dedieu JF, et al. RGD inclusion in the hexon monomer provides adenovirus type 5-based vectors with a fiber knob-independent pathway for infection. $\mathcal{F}$ Virol 1999;73:5156-61.

55 Wathway for infection. Firol 1999;73:5156-61. 70 7:110-14.

56 Roelvink PW, Lee GM, Einfeld DA, et al. Identification of a conserved receptor-binding site on the fiber proteins of CAR-recognizing adenoviridae. Science 1999;286:1568-71.

57 Bewley MC, Springer K, Zhang YB, et al. Structural analysis of the mechanism of adenovirus binding to its human cellular receptor, CAR. Science 1999;286:1579-83.
58 Kirby I, Davison E, Beavil AJ, et al. Identification of contact residues and definition of the CAR-binding site of adenovirus type 5 fiber protein. $f$ Virol 2000;74:2804-13

59 Douglas JT, Miller CR, Kim M, et al. A system for the propagation of adenoviral vectors with genetically modified receptors. Nat Biotechnol 1999;17:470-5.

60 Von Seggern DJ, Huang S, Fleck SK, et al. Adenovirus vector pseudotyping in fiber-expressing cell lines: Improved transduction of Epstein-Barr virustransformed B cells. F Virol 2000;74:354-62. 